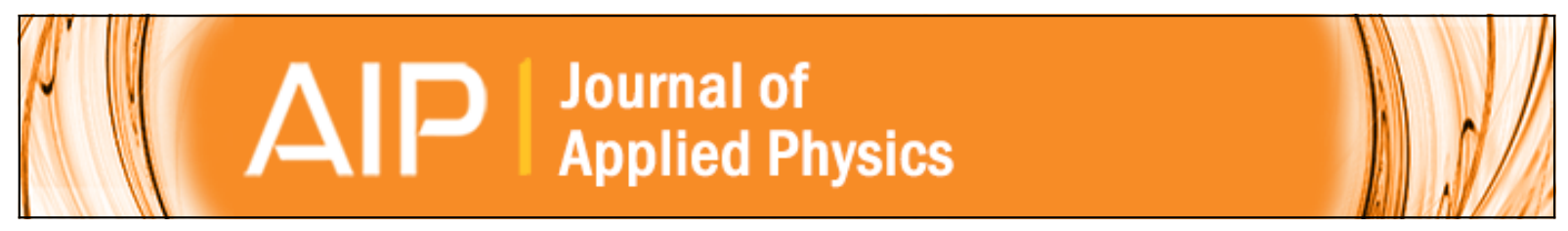

\title{
Generalized analysis of quasi-steady-state and transient decay open circuit voltage measurements
}

Mark J. Kerr, Andres Cuevas, and Ronald A. Sinton

Citation: Journal of Applied Physics 91, 399 (2002); doi: 10.1063/1.1416134

View online: http://dx.doi.org/10.1063/1.1416134

View Table of Contents: http://scitation.aip.org/content/aip/journal/jap/91/1?ver=pdfcov

Published by the AIP Publishing

\section{Articles you may be interested in}

Anomalously high lifetimes measured by quasi-steady-state photoconductance in advanced solar cell structures Appl. Phys. Lett. 103, 243902 (2013); 10.1063/1.4840337

Accurate determination of minority carrier mobility in silicon from quasi-steady-state photoluminescence J. Appl. Phys. 113, 213705 (2013); 10.1063/1.4807926

Minority carrier lifetime in silicon wafers from quasi-steady-state photoluminescence

Appl. Phys. Lett. 97, 092109 (2010); 10.1063/1.3485216

Effect of dislocations on open circuit voltage in crystalline silicon solar cells

J. Appl. Phys. 100, 093708 (2006); 10.1063/1.2360773

Generalized analysis of quasi-steady-state and quasi-transient measurements of carrier lifetimes in semiconductors

J. Appl. Phys. 86, 6218 (1999); 10.1063/1.371633

\section{AIP $\mid$ APL Photonics \\ APL Photonics is pleased to announce Benjamin Eggleton as its Editor-in-Chief}

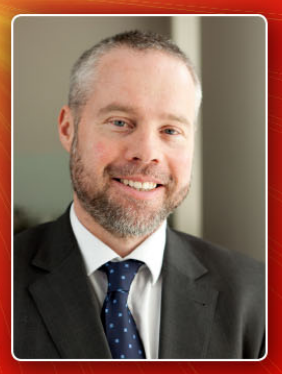




\title{
Generalized analysis of quasi-steady-state and transient decay open circuit voltage measurements
}

\author{
Mark J. Kerra) and Andres Cuevas \\ Centre for Sustainable Energy Systems, Department of Engineering, Australian National University, \\ Canberra ACT 0200, Australia \\ Ronald A. Sinton \\ Sinton Consulting Inc., 1132 Green Circle, Boulder, Colorado 80305
}

(Received 24 May 2001; accepted for publication 10 September 2001)

\begin{abstract}
The current-voltage characteristics of solar cells and photodiodes can be determined by measuring the open-circuit voltage as a function of a slowly varying light intensity. This article presents a detailed theoretical analysis and interpretation of such quasi-steady-state $V_{\text {oc }}$ measurements $\left(\mathrm{Qss} V_{\mathrm{oc}}\right)$. The ability of this analysis to accurately obtain the true steady-state device characteristics even in the case of high lifetime, high resistivity silicon devices is demonstrated experimentally. The Qss $V_{\text {oc }}$ technique can be used to determine the minority carrier lifetime, and the new generalized analysis is required to do this accurately. An important outcome is that solar cell and diode device characteristics can be obtained from measurements of either the photoconductance or the open-circuit voltage, even using transient techniques. (C) 2002 American Institute of Physics.
\end{abstract}

[DOI: $10.1063 / 1.1416134]$

\section{INTRODUCTION}

The recombination properties of semiconductor devices strongly affect their electrical performance. Experimentally measuring these recombination properties in a simple and easy way has been significantly facilitated by the introduction of quasi-steady-state (Qss) techniques. ${ }^{1,2}$ The basis for them is that the steady-state behavior of the device can be accurately determined even if the measuring conditions are not fully steady state. If the rate of change of the excitation is much slower than the recombination rate, the device will practically be in steady-state conditions. The interest of varying the excitation is that a range of different steady-state operating points can be conveniently explored. The Qss approach has already proven to be very powerful in the measurement of minority carrier lifetimes in silicon wafers. ${ }^{1}$ In the quasi-steady-state photoconductance (QssPC) technique, a simple flashlamp is used to produce a slowly varying illumination and the resulting time dependence of the excess photoconductance of the sample is measured. Analysis of the data gives the minority carrier effective lifetime as a function of injection level. The ease with which the lifetime can be measured over a vast range of carrier injection conditions offers a multitude of possibilities to investigate the different recombination mechanisms that can be present in a silicon wafer. $^{3}$

The Qss approach has also been applied to measure the open-circuit voltage of solar cells as a function of the incident light intensity. ${ }^{4}$ Again, a monotonically varying illumination from a flashlamp is used to produce a voltage versus illumination curve in a fraction of a second. This quasisteady-state open-circuit voltage method $\left(\mathrm{Qss} V_{\text {oc }}\right)$ has important advantages over the classic $I_{\mathrm{sc}}-V_{\mathrm{oc}}$ technique used in

a)Electronic mail: mark.kerr@faceng.anu.edu.au the past as the primary method to measure the characteristics of the $p n$ junction free from series resistance effects. Besides an almost direct measurement of the $I-V$ characteristics, the Qss $V_{\text {oc }}$ technique can also provide an insight into carrier recombination and an alternative path to determine the minority carrier lifetime. It nicely complements the QssPC photoconductance technique by further broadening the range of measurable injection levels while avoiding carrier trapping effects. ${ }^{5}$

Since the lifetime of silicon samples can be anywhere from about $1 \mu$ s to more than $10 \mathrm{~ms}$, it is important to choose the variable light source appropriately. In most practical cases a flashlamp with a dimming rate of $\sim 2 \mathrm{~ms}$ is adequate. This is particularly true for finished devices on low resistivity silicon, because the highly doped regions and metal contacts usually bring the effective lifetime to values below $200 \mu \mathrm{s}$, in comparison to which a $2 \mathrm{~ms}$ flash is seen by the device as a steady-state excitation. It is, nevertheless, possible to analyze the data in a way that accommodates the possible different time dependencies of the light excitation and the excess carrier density in the semiconductor. Such generalized analysis has been applied to improve the accuracy of QssPC measurements. ${ }^{6}$ A similar analysis of Qss voltage measurements is presented in this article. This corrected analysis is particularly important for solar cells made on high resistivity, high lifetime silicon, and we have used such devices to demonstrate the method experimentally.

The extended analysis of time-dependent measurements of the excess carrier density under illumination by either conductance or voltage techniques goes beyond pure mathematical rigor. Applying it to the special case where the light excitation is abruptly terminated and the transient decay of the photoconductance or the voltage is recorded with time is very important, since the photoconductance decay (PCD) 
and open-circuit voltage decay (OCVD) methods have historically been, and still are, widespread. As shown in this article, it is interesting to realize that, even if the light intensity and the electric current are zero, it is still possible to obtain the characteristic curves of the device from PCD and OCVD measurements.

\section{GENERALIZATION OF EFFECTIVE LIFETIME MEASUREMENTS}

\section{A. Previous work}

Three operating regimes for the light excitation can be identified. The first involves an abrupt cessation of the illumination and subsequent analysis of the sample's excess photoconductance without any illumination. This is the traditional transient photoconductance technique, which can be easily applied to high lifetime samples with only simple electronics. The second regime is the steady-state illumination, which has also been historically used, although not as frequently as the PCD method. In the third regime the intensity of the illumination varies monotonically with time, which is the basis of the quasi-steady-state method. Obviously the first two regimes are extreme cases of the third, which therefore represents the general case. Proper analysis of all the different possibilities requires use of the continuity equation for the excess minority carriers:

$$
\frac{\partial \Delta n}{\partial t}=G_{b}(t, x)-U_{b}(t, x)+\frac{1}{q} \frac{d J_{n}}{d x} .
$$

Where $G_{b}(t, x)$ and $U_{b}(t, x)$ are the photogeneration rate and recombination rate in the bulk, $\Delta n$ the excess minority carrier density, and $J_{n}$ the electron current density. For regime 1: $G_{b}(t, x)=0$, while for regime 2: $\partial \Delta n / \partial t=0$. Furthermore, the transport term reduces to surface recombination terms when Eq. (1) is integrated over the sample width because the sample is in open-circuit conditions. Nagel et al. ${ }^{6}$ generalized the analysis procedure to define an effective minority carrier lifetime regardless of the flashlamp characteristics by combining the bulk and surface recombination rates into an effective recombination rate $\left(U_{\text {eff }}\right)$ and not dropping any of the terms in Eq. (1):

$$
\tau_{\text {eff }}=\frac{\Delta n_{a \nu}(t)}{G_{a \nu}(t)-\frac{\partial \Delta n_{a \nu}(t)}{\partial t}} .
$$

To understand the physical meaning underlying this generalized definition of lifetime, it should be realized that the recombination rate occurring within an illuminated sample can be affected by both the photogeneration rate at that instance, as well as the history of the sample (through the timedependent carrier density, $\partial \Delta n / \partial t$ ). What is not obvious from the generalized lifetime definition of Nagel et al. is how to determine other device parameters, like the true steady-state open-circuit voltage or current-voltage characteristic of a solar cell, from QssPC or transient decay measurements.

\section{B. Determination of implied open-circuit voltage vs light intensity curves from photoconductance measurements}

Effectively, the correction of Nagel et al. is equivalent to defining the denominator of Eq. (2) as a net generation rate $\left(G_{\text {net }}\right)$ that incorporates the actual photogeneration $\left(G_{a v}\right)$ and the carrier density history:

$$
G_{\mathrm{net}}=G_{a \nu}-\frac{\partial \Delta n_{a \nu}}{\partial t} .
$$

In the context of characterizing the recombination parameters of solar cells and/or their precursors under real conditions (i.e., under true steady-state conditions), the concept of net generation can be greatly expanded. The simplest extension of the net generation concept is for obtaining implied open-circuit voltage versus light intensity curves from photoconductance based lifetime measurements. ${ }^{2}$ Under the quasi-steady-state assumption, the actual illumination level ( $I_{\text {light }}$, in suns) can be plotted against an implied open-circuit voltage ( $\left.V_{\text {oc,imp }}\right)$ using the relationship between the $p n$ product under nonequilibrium conditions to its equilibrium value $n_{i}^{2}$ at the boundary of the junction space charge region (for a $p$-type wafer $n \approx \Delta n$ and $p \approx N_{A}+\Delta n$ ):

$$
n p \approx \Delta n(0)\left[N_{A}+\Delta n(0)\right]=n_{i}^{2} \exp \frac{q V_{\mathrm{oc}}}{k T} .
$$

We know however that the actual illumination level $\left(I_{\text {light }}\right)$, and therefore the actual photogeneration rate $\left(G_{a v}\right)$, does not necessarily account for all the recombination events occurring within the sample. The net generation rate $\left(G_{\text {net }}\right)$, as defined in Eq. (3), needs to be used to determine the equivalent, steady-state illumination level $\left(I_{\text {net }}\right)$ that would need to be used to obtain the same total recombination rate. This can be expressed in unit of suns $\left(1 \operatorname{sun}=1 \mathrm{~kW} / \mathrm{m}^{2}\right)$ via:

$$
I_{\text {net }}=\frac{W G_{\text {net }}}{f_{\text {abs }} N_{\text {ph|1 sun }}},
$$

where $W$ is the sample width, $f_{\text {abs }}$ is the optical absorption fraction of the sample to take into account reflection and absorption losses and $N_{\mathrm{ph} \mid 1 \text { sun }}$ is the density of photons in solar light with an irradiance of 1 sun $\left(1 \mathrm{~kW} / \mathrm{m}^{2}\right)$. Alternatively, $f_{\mathrm{abs}} N_{\mathrm{ph} \mid 1 \text { sun }}$ can be replaced with an estimated or measured short-circuit current density $\left(J_{\mathrm{sc}}\right)$ under one sun standard illumination.

An example of an implied open-circuit voltage versus light intensity curve both before the correction $\left(I_{\text {light }}-V_{\text {oc,imp }}\right)$ and after the correction $\left(I_{\text {net }}-V_{\text {oc,imp }}\right)$ is given in Fig. 1. The flashlamp used showed an approximately exponential reduction in light intensity over time with a decay time of $\approx 2 \mathrm{~ms}$. The sample being measured is a high lifetime solar cell precursor consisting of a phosphorus diffused, high resistivity silicon substrate passivated with high quality TCA based silicon oxide on the front and rear. It can be seen that the uncorrected data are actually quite discontinuous, particularly at lower light levels, while the corrected data produce a smooth, continuous curve, which is physically what would be expected. Furthermore, by comparing the uncorrected and corrected quasi-steady-state data it can be seen 


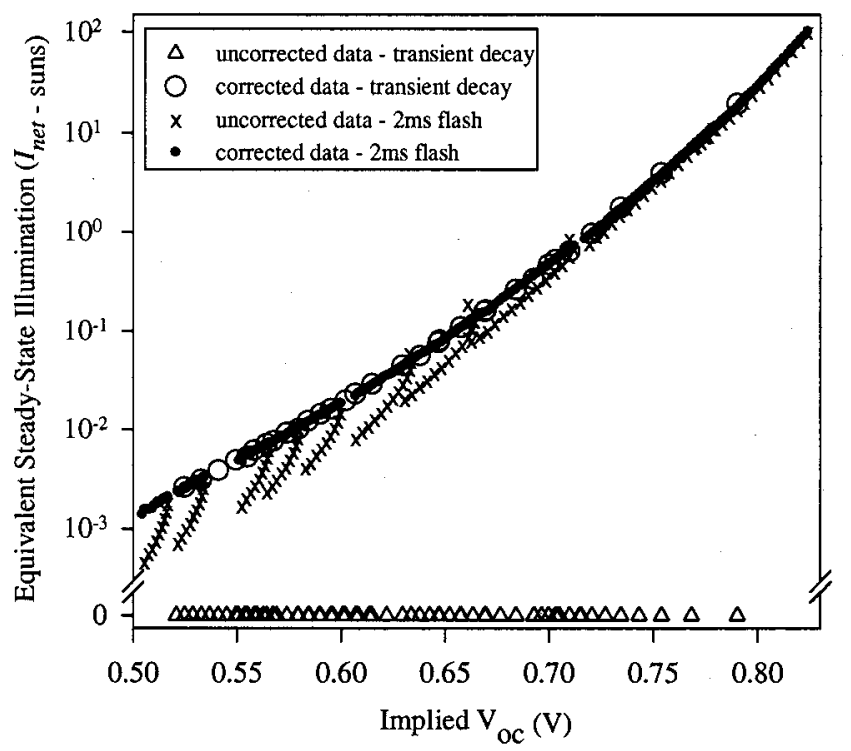

FIG. 1. Corrected and uncorrected implied open-circuit voltage vs light intensity curves for a solar cell precursor from photoconductance measurements. A flashlamp with a decay time of $\sim 2 \mathrm{~ms}$ has been used as well as in transient mode where there is no actual illumination of the sample during the measurement. The vertical axis is determined from $G_{a v}$ for the uncorrected data and from $G_{\text {net }}$ for the corrected data using Eq. (5).

that the correction starts to become significant at light intensities below $\approx 1$ sun for this sample. The effect of the correction is to provide a more realistic determination of the electronic properties of the solar cell substrate. That is, the uncorrected data overestimate the quality of the solar cell substrate by overpredicting the $V_{\mathrm{oc}}$.

An interesting, not so obvious consequence of using the net generation concept is that transient photoconductance decay measurements can be used to determine implied opencircuit voltage versus net light intensity curves even though the actual light intensity is zero. Included in Fig. 1 are corrected and uncorrected implied open-circuit voltage data when the lifetime is measured by the transient method. It can be seen that the two corrected curves agree very well, demonstrating the validity of the approach.

The agreement between PCD and QssPC data can be exploited to determine, or verify, the optical properties of the wafer, that is the factor $f_{\text {abs }}$ in Eq. (5). Furthermore, the standard diode analysis and interpretation can be made on the $I_{\text {light }}-V_{\text {oc,imp }}$ curves, thus relating the injection level dependent effective lifetime of the sample to ideality factor at a given open-circuit voltage. For example, the corrected $I_{\text {net }}-V_{\text {oc,imp }}$ curve in Fig. 1 shows nonideal diode behavior at lower voltages for this sample $(n \neq 1)$ due to injection level dependent surface recombination velocity and bulk lifetime.

\section{Determination of photovoltaic $I-V$ curves from photoconductance measurements}

An alternative transformation of implied open-circuit voltage versus light intensity data is an implied photovoltaic $I-V$ curve. ${ }^{4}$ This transformation uses the superposition principle to present the data in the familiar $I-V$ format. In this

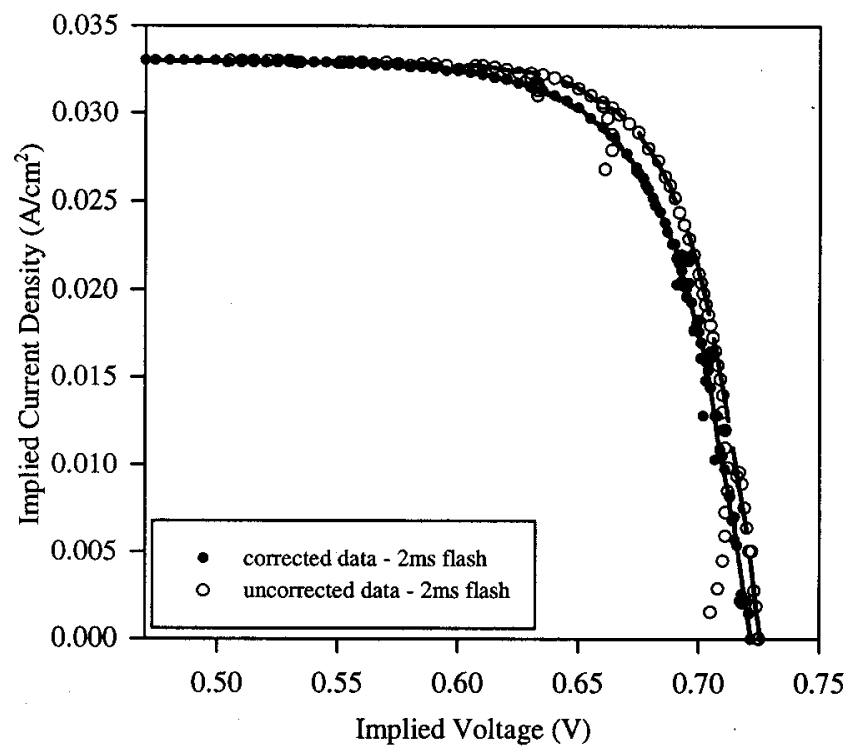

FIG. 2. Implied photovoltaic $I-V$ curves determined from the corrected and uncorrected photoconductance data (both QssPC and PCD) for the solar cell precursor of Fig. 1. The effect of the correction is to lower the cell's opencircuit voltage and fill factor.

format, the customary interpretation of fill factor, conversion efficiency (without series resistance) and shunt resistance can be easily made, even though no electric current is actually being measured. An implied terminal current $\left(J_{\text {imp }}\right)$ is determined for each open-circuit voltage from the net illumination level $\left(I_{\text {net }}\right)$ and an estimated short-circuit current density $\left(J_{\mathrm{sc}}\right)$ under one sun illumination. Indeed, an upper limit to the short-circuit current density $\left(J_{\mathrm{sc}, \max }\right)$ has already been established in determining the optical absorption fraction of the sample $\left(J_{\mathrm{sc}, \max }=q f_{\mathrm{abs}} N_{\mathrm{ph} \mid 1 \text { sun }}\right)$, with the equality relationship holding when the cell has $100 \%$ internal quantum efficiency at all wavelengths:

$$
J_{\text {imp }}=J_{S C}\left(1-I_{\text {net }}\right) \leqslant q f_{\text {abs }} N_{\text {ph } \mid 1 \text { sun }}\left(1-I_{\text {net }}\right) .
$$

An implied photovoltaic $I-V$ curve is then obtained by plotting the implied open-circuit voltage $\left(V_{\text {oc,imp }}\right)$ against the implied terminal current $\left(J_{\text {imp }}\right)$. This is shown in Fig. 2 for the uncorrected and corrected data ( $\sim 2 \mathrm{~ms}$ flashlamp) from Fig. 1 , by assuming a $J_{\mathrm{sc}}=33 \mathrm{~mA} / \mathrm{cm}^{2}$. The correction lowers the 1-sun $V_{\text {oc }}$ from $725.0 \mathrm{mV}$ down to $721.6 \mathrm{mV}$ and the recombination limit to the fill factor is reduced from $85.7 \%$ to $83.6 \%$. The upper limit to the cell efficiency is reduced from $20.4 \%$ down to $19.9 \%$ and the device appears to be shunt free (note the expanded scale of the $x$ axis). It should be noted that these are upper limits for an actual solar cell from this substrate, as the final device would also include contact recombination, series resistance effects and shading losses. Importantly, we could have equally determined the photovoltaic $I-V$ curve of this sample from the corrected transient data in Fig. 1, thanks to the generalized definition of $I_{\text {net }}$. 


\section{GENERALIZATION OF OPEN-CIRCUIT VOLTAGE MEASUREMENTS}

\section{A. Introduction}

The objective of a Qss $V_{\text {oc }}$ measurement is to obtain the diode characteristics of a finished solar cell. These characteristics come about by simply plotting the actual open-circuit voltage of the device as a function of the light intensity. We are seeking the dc, or steady-state characteristics of the device, that is, the dc value of $V_{\mathrm{oc}}$ that corresponds to a given value of steady-state light intensity. Analogous to the discussion in Sec. II, the actual illumination level at the time does not necessarily account for all the carriers in the sample. In particular, it does not necessarily account for all the carriers at the boundary of the space charge region, which in-turn, determine the open-circuit voltage. Again, we need to consider the photogeneration history of the sample by using the net generation rate of Eq. (3). This can be achieved by solving the quadratic expression of Eq. (4) to determine $\Delta n$ (only the positive root has a physical meaning):

$$
\Delta n=\frac{\sqrt{N_{A}^{2}+4 n_{i}^{2} \exp \frac{q V_{\text {oc }}}{k T}}-N_{A}}{2},
$$

where differentiating gives

$$
\frac{\partial \Delta n}{\partial t}=\frac{q n_{i}^{2} \exp \frac{q V_{\mathrm{oc}}}{k T}}{k T \sqrt{N_{A}^{2}+4 n_{i}^{2} \exp \frac{q V_{\mathrm{oc}}}{k T}}} \frac{\partial V_{\mathrm{oc}}}{\partial t},
$$

which can be substituted into Eq. (3) and then into Eq. (5) to obtain the equivalent, steady-state illumination level $\left(I_{\text {net }}\right)$ that needs to be plotted against the measured $V_{\text {oc }}$. This procedure outlines the generalization of $V_{\mathrm{oc}}$ measurements as a function of light intensity and time.

\section{B. Experimental illustration of the correction}

The open-circuit voltage as a function of light intensity was measured for a low recombination solar cell made at the ANU on a $90 \Omega \mathrm{cm}$ phosphorus-doped silicon wafer. The cell had full area boron and phosphorus diffusions at the front and rear of the cell, respectively, essentially making it a onedimensional device. The high open-circuit voltage at one sun, $677 \mathrm{mV}$, testifies the quality of the device $\left(J_{\mathrm{sc}}\right.$ $\left.=32.2 \mathrm{~mA} / \mathrm{cm}^{2}, \mathrm{FF}=0.781, \eta=17.1 \%\right)$. It also means that the excess carrier density is very high, which adds to the problem of maintaining steady-state conditions during a time-dependent measurement.

Four different modes of a flashlamp were used to illustrate the generalized analysis of $\mathrm{Qss} V_{\mathrm{oc}}$ measurements. Along with the transient mode, the decay times of the flashlamps were $\approx 0.35, \approx 2$, and $\approx 4 \mathrm{~ms}$. The $0.35 \mathrm{~ms}$ flashlamp has been included to demonstrate the robustness of the correction. It would not typically be used for quasisteady-state measurements. Neutral density filters were used to vary the intensity of light incident on the solar cell to between 100 suns and 0.001 suns.

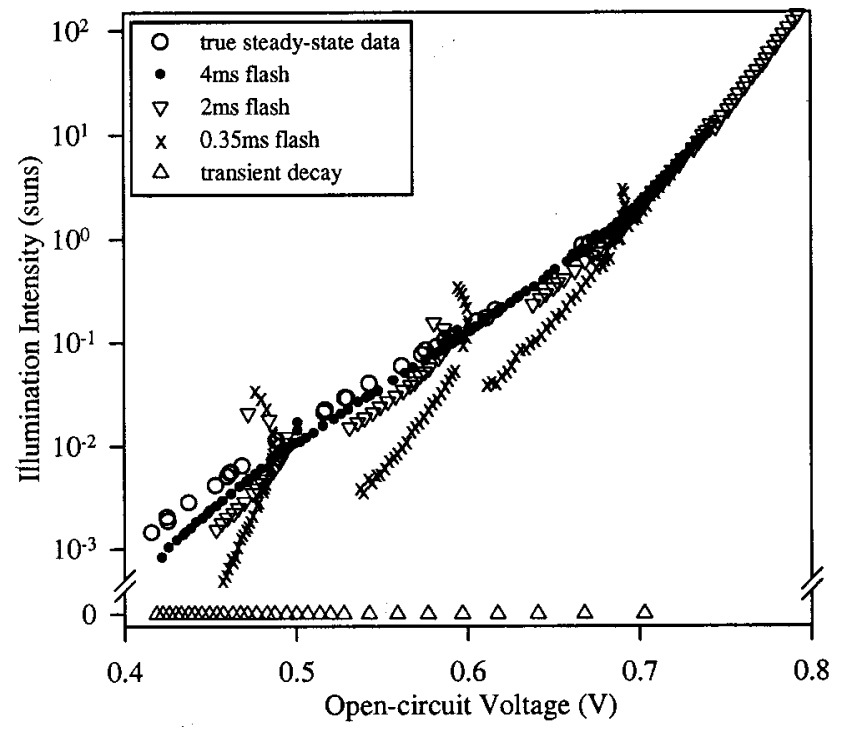

FIG. 3. Uncorrected open-circuit voltage vs light intensity curves for a low recombination solar cell. The illumination modes for the solar cell include transient illumination as well as flashlamp decay times of $\approx 0.35, \approx 2$, and $\approx 4 \mathrm{~ms}$. True steady-state measurements have been included for comparison.

Uncorrected $I_{\text {light }}-V_{\text {oc }}$ curves for the device are plotted in Fig. 3 along with true steady-state measurements obtained by attenuating the illumination from a solar simulator with several different neutral density filters and placing the device subsequently in open and short circuit conditions. Consistent with the discussion in Sec. II B, the uncorrected curves are quite discontinuous. Most importantly, the predicted performance of the device would vary greatly depending on which flashlamp was used and would be significantly different from the true steady-state performance. Even for the $4 \mathrm{~ms}$ flashlamp, there is a noticeable difference between the true steady-state measurements and the uncorrected $I_{\text {light }}-V_{\mathrm{oc}}$ data at illumination levels below one sun.

The corrected $I_{\text {net }}-V_{\text {oc }}$ curves for the device are plotted in Fig. 4. It can be seen that using the generalized analysis removes the discontinuities in the curves for all the illumination modes, but most importantly, all the curves now overlap with each other and with the true steady-state measurements. We have also demonstrated the accuracy of the generalized analysis for high efficiency devices made on low resistivity silicon, ${ }^{7}$ further supporting the validity of our generalized analysis for $\mathrm{Qss} V_{\text {oc }}$ measurements.

\section{Determination of the lifetime from $V_{\text {oc }}$}

An important application of the $\mathrm{Qss} V_{\mathrm{oc}}$ technique is to determine the effective minority carrier lifetime in finished devices and it is a useful accompaniment to the more common OCVD technique. ${ }^{8}$ It is again important to account for the photogeneration history of the device. The effective lifetime can be determined by substituting Eqs. (7) and (8) into Eq. (2), giving the generalized definition of the effective minority carrier lifetime from the open-circuit voltage: 


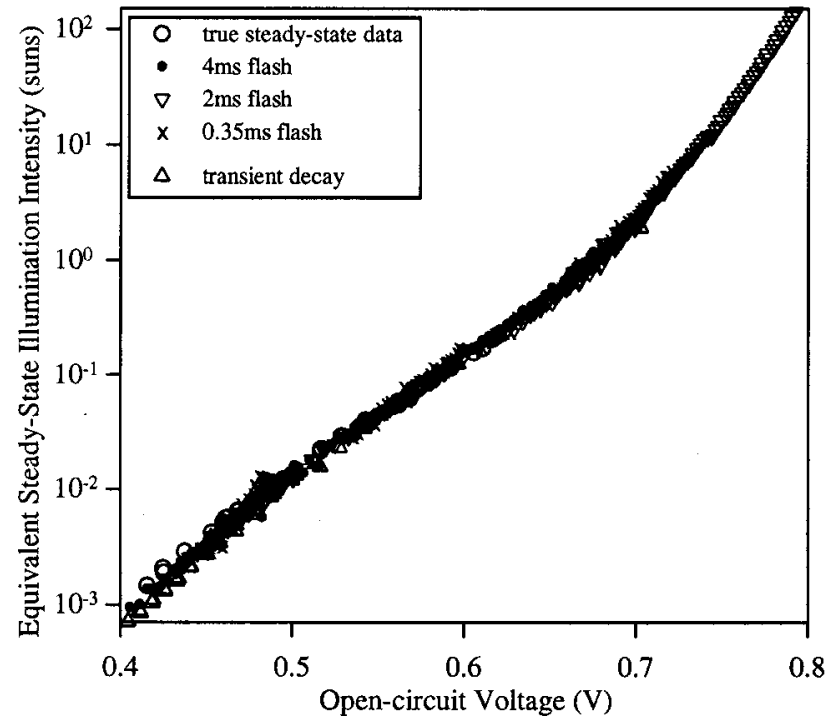

FIG. 4. Corrected open-circuit voltage vs light intensity curves for a low recombination solar cell using four different illumination conditions. The corrected curves all essentially overlap with the true steady-state measurements.

$$
\tau_{\mathrm{eff}}=\frac{\sqrt{N_{A}^{2}+4 n_{i}^{2} \exp \frac{q V_{\mathrm{oc}}}{k T}}-N_{A}}{2\left(G_{a \nu}-\frac{q n_{i}^{2} \exp \frac{q V_{\mathrm{oc}}}{k T}}{\left.k T \sqrt{N_{A}^{2}+4 n_{i}^{2} \exp \frac{q V_{\mathrm{oc}}}{k T}} \frac{\partial V_{\mathrm{oc}}}{\partial t}\right)},\right.}
$$

Which, for the case of low level injection, can be simplified to a more familiar form that is easily identifiable with the OCVD lifetime when the generation rate is zero: ${ }^{8}$

$$
\tau_{\mathrm{eff}}=\frac{\Delta n}{G_{a \nu}-\frac{q \Delta n}{k T} \frac{\partial V_{\mathrm{oc}}}{\partial t}} .
$$

The lifetime can thus be easily calculated from the measured voltage, its time derivative and the actual illumination level. Figure 5 shows the effective lifetime corresponding to a high efficiency PERL cell fabricated at Fraunhofer Institute of Solar Energy Systems on a $22 \Omega \mathrm{cm}$ gallium-doped silicon substrate $\left(V_{\mathrm{oc}}=680 \mathrm{mV}, \quad J_{\mathrm{sc}}=39.5 \mathrm{~mA} / \mathrm{cm}^{2}, \quad \mathrm{FF}=0.793, \quad \eta\right.$ $=21.2 \%$ ). Minority carrier lifetimes in excess of $1 \mathrm{~ms}$ have been independently measured for these wafers by photoconductance techniques. It can be seen that the lifetime determined using the generalized analysis of Eq. (9) has a maximum value of $\approx 1.1 \mathrm{~ms}$ at an injection level of 3 $\times 10^{14} \mathrm{~cm}^{-3}$.

Included in Fig. 5 are the effective lifetime values determined for flashlamps with decay times of 2 and $4 \mathrm{~ms}$ but neglecting the device's photogeneration history. The maximum lifetime depends on the decay time of the flashlamp, with a maximum of $\approx 2.2$ and $\approx 1.6 \mathrm{~ms}$ for a 2 and $4 \mathrm{~ms}$

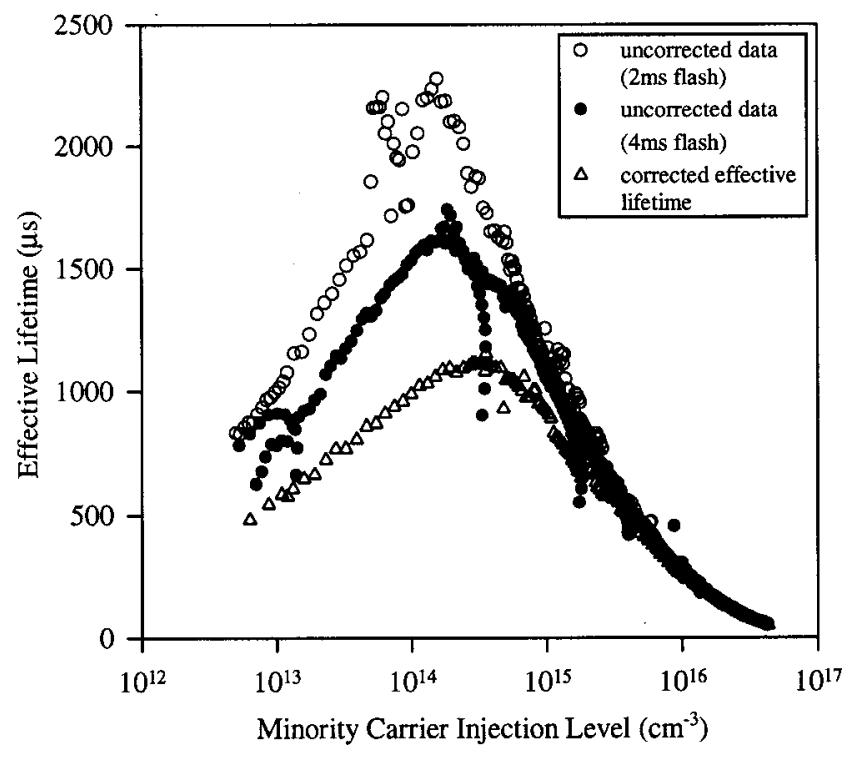

FIG. 5. Minority carrier lifetime data corresponding to corrected and uncorrected $I_{\text {light }}-V_{\text {oc }}$ curves for a high efficiency PERL cell. The flashlamps used for illuminating the cell had decay times of $\approx 2$ and $\approx 4 \mathrm{~ms}$.

decay constant flashlamp, respectively. Clearly, the use of the generalized analysis is required to accurately determine the effective lifetime.

\section{Determination of the photovoltaic $I-V$ curve from $V_{\text {oc }}$}

Open-circuit voltage versus light intensity data can easily be converted into a photovoltaic $I-V$ curve as discussed in Sec. II C. For the solar cell characterized in Fig. 4, it is clear that the corrected data all overlap regardless of the flashlamp characteristics. It thus follows that the photovoltaic $I-V$ curves obtained from these data will also overlap, regardless of the flashlamp characteristics. The extreme case is shown in Fig. 6, where the photovoltaic $I-V$ curve determined using true steady-state illumination $\left(J_{\mathrm{sc}}-V_{\mathrm{oc}}\right.$ data $)$ and using transient illumination (OCVD) are compared. It can be seen that both agree very well.

Also included in Fig. 6 is the 1-sun $I-V$ curve (obtained by changing the voltage bias and measuring the current), which is in good agreement with the two photovoltaic $I-V$ curves. As 1-sun $I-V$ curves include series resistance effects but photovoltaic $I-V$ curves do not, it is concluded that this cell has low series resistance. This is to be expected as the cell has a diffused region at the front and rear surface. Importantly, it is clear that transient decay (OCVD) can be successfully used to predict upper limits on the performance of solar cells under steady-state conditions using the generalized $I_{\text {net }}$.

\section{DISCUSSION}

It has already been pointed out that using the net generation rate leads to a more realistic determination of solar cell properties (lower $V_{\mathrm{oc}}$ and fill-factor) from Qss $V_{\mathrm{oc}}$ and QssPC measurements. The magnitude of the correction is largest when the effective lifetime of the sample is of the same 


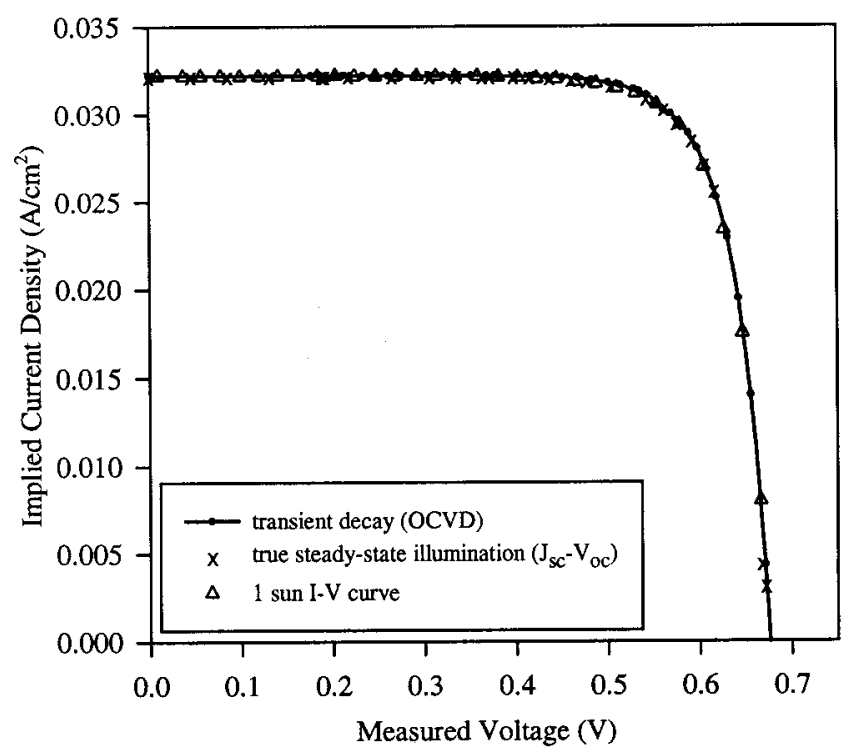

FIG. 6. Photovoltaic $I-V$ curve, for the solar cell characterized in Fig. 4, using transient decay (OCVD) and steady-state illumination $\left(J_{\mathrm{sc}}-V_{\mathrm{oc}}\right)$. The 1 -sun $I-V$ curve is included for comparison.

order, or longer than the decay time of the flashlamp used. In this article we have demonstrated the generalized analysis procedure using high efficiency and high lifetime solar cells. For the majority of industrial solar cells the effective lifetime is quite small $(10-50 \mu \mathrm{s})$ and is much less than the decay time of the typical flashlamps used for these measurements (decay time of $\approx 2-4 \mathrm{~ms}$ ). Under these conditions, the effect of the generalized analysis presented here to determine $I-V$ characteristics is small. The errors in determining the effective lifetime can be significantly greater. ${ }^{7}$

Although the experimental measurements plotted throughout this article have been focused on illustrating the correction to the photogeneration rate, we have observed capacitive effects at very low voltages. Junction capacitance has traditionally been one of the major drawbacks of the OCVD method, since it dominates the decay of the voltage at low carrier densities. ${ }^{9}$ The $\mathrm{Qss} V_{\text {oc }}$ technique is not totally impervious to these effects, but they only become significant at much lower voltages. The corresponding excess carrier densities are extremely low (of the order of the intrinsic carrier density), and both the recombination and generation (less than one thousand of one sun) rates are very small. In such circumstances, the capacitive effect of the $p n$ junction becomes important. We have verified that the distortion produced by this capacitance is directly related to the light source used: it is highest for the transient measurement, less severe for the $0.35 \mathrm{~ms}$ flash, and moderate to negligible for the 2 and 4 ms flashes. This indicates that the effect can be practically eliminated by using a still slower flash. Alternatively, the capacitance could be determined from the transient OCVD measurement and then its value used to correct the $\mathrm{Qss} V_{\text {oc }}$ measurement. Equation (1) would need to be modified to add a term due to junction capacitance. Additional physical mechanisms, such as a shunt resistance, could also be added to the analysis.

Finally, while we have shown that both Qss and transient techniques can be accurately used to evaluate device performance, there are some differences. ${ }^{7}$ If the main objective is to determine the steady-state characteristics of the device (the steady-state recombination rate, or the dc currentvoltage characteristics), then the experimental conditions should preferably approach the steady state as much as possible. In the case of solar cells, testing under illumination is clearly closer to the intended operating conditions than testing their transient behavior in the dark.

\section{CONCLUSIONS}

The physical concept of a net photogeneration rate as the sum of the actual photogeneration and the derivative of the excess carrier density with time has been introduced. This leads to a generalized analysis procedure for illumination versus open-circuit voltage characteristic curves of solar cells. In this article we have shown that the $\mathrm{Qss} V_{\text {oc }}$ technique can be accurately applied, even to high lifetime devices, for a given time-dependent light source when the generalized analysis is used. It is also permitted to obtain net illumination versus voltage curves even for transient decay measurements of the photoconductance or the open-circuit voltage, a feature previously reserved exclusively to steady-state or Qss techniques.

\section{ACKNOWLEDGMENTS}

This work has been funded by the Australian Research Council. The authors also thank S. Glunz and S. Rein from the Fraunhofer Institute of Solar Energy Systems, for the use of the solar cell used in Fig. 5.

\footnotetext{
${ }^{1}$ R. A. Sinton and A. Cuevas, Appl. Phys. Lett. 69, 2510 (1996).

${ }^{2}$ A. Cuevas and R. A. Sinton, Prog. Photovoltaics 5, 79 (1997).

${ }^{3}$ A. Cuevas, M. Stocks, D. Macdonald, M. Kerr, and C. Samundsett, IEEE Trans. Electron Devices ED-46, 2026 (1999).

${ }^{4}$ R. A. Sinton and A. Cuevas, in A Quasi-Steady-State Open-Circuit Voltage Method for Solar Cell Characterization, Proceedings of the 16th European Photovoltaic Solar Energy Conference, Glasgow, Scotland, 2000, pp. $1152-1155$.

${ }^{5}$ D. Macdonald and A. Cuevas, Prog. Photovoltaics 8, 363 (2000).

${ }^{6}$ H. Nagel, C. Berge, and A. Aberle, J. Appl. Phys. 86, 6218 (1999).

${ }^{7}$ M. J. Kerr, Ph.D. thesis Australian National University, 2001.

${ }^{8}$ D. K. Schroder, Semiconductor Material and Device Characterization (Wiley, New York, 1990).

${ }^{9}$ J. E. Mahan and D. L. Barnes, Solid-State Electron. 24, 989 (1981).
} 